\title{
Nalbuphine Attenuates Microglial Activation and Inhibits Morphine-Induced Scratching via Regulation of PKC $\delta$ and p38 Signaling in Mice
}

\section{Yang Tang}

Guangzhou Medical University Second Affiliated Hospital

Nan Qi Li

Sun Yat-sen University First Affiliated Hospital

Si Ting Huang

Guangzhou Medical University Second Affiliated Hospital

Zhe Peng

Guangzhou Medical University Second Affiliated Hospital

Li Wan ( $\nabla$ wanli5000cn@163.com )

https://orcid.org/0000-0002-8729-4018

\section{Research}

Keywords: itch, KOR, microglia, morphine, nalbuphine, p38 MAPK, PKC

Posted Date: May 13th, 2020

DOI: https://doi.org/10.21203/rs.3.rs-28612/v1

License: (c) (1) This work is licensed under a Creative Commons Attribution 4.0 International License. Read Full License 


\section{Abstract}

Morphine-induced scratching (MIS) is a common issue in patients receiving clinical postoperative morphine intrathecal injections. The kappa opioid receptor (KOR) agonist nalbuphine is known to prevent and reduce MIS, but the underlying mechanism has remained unclear. Here, we found that protein kinase $\mathrm{C}(\mathrm{PKC} \beta)$ in the spinal cord dorsal horn was co-expressed with ionized calcium binding adapter molecule 1 (Iba1) and increased after morphine intrathecal (i.t.) injections in mice. Although knockdown PKC $\beta$ inhibited microglial activation and attenuated MIS, but nalbuphine can blocked MIS by promoting PKC $\delta$ expression rather than $\mathrm{PKC} \beta$ expression, suggesting that antipruritic effect of nalbuphine is due to activation of the KOR/PKC $\delta$ pathway and that MIS is both $\mathrm{PKC} \beta$-dependent and closely related to microglial activation. Moreover, we also found that PKC $\delta$ could retroegulation microglial activation, thereby reversing nalbuphine's inhibition of MIS and resulting in increased itching behaviors. Notably, microglial activation is required for p-p38 signaling in MIS. These data together suggest that nalbuphine activates KOR, so as to inducing the activation of $\mathrm{PKC}$, which may in turn retroegulation microglial function and decrease phosphorylate $\mathrm{p38}$, ultimately inhibiting MIS. Our research therefore indicates that a previously unknown KOR-PKC $\delta$-microglia-p38 pathway in the spinal cord may underlie nalbuphine's antipruritic effect.

\section{Introduction}

Pruritus, or itching, is defined as an unpleasant sensation that elicits the desire or reflex to scratch[1]. As an evolutionarily conservative behavior, itching reminds people to avoid harmful environmental stimuli. However, not every itch can be prevented, and itching sensation is often unpleasant. Itching induced by opioid intrathecal injections is an uncomfortable sensation that occurs during postoperative analgesia, with a reported incidence of $18.7 \%$ [2]. Although intrathecal injections of morphine can effectively block postoperative pain, opioid-induced pruritus also significantly influences patients' analgesia experience and limits the successful application of opioids[3].

Nalbuphine is a kappa opioid receptor (KOR) agonist and mu opioid receptor antagonist; it has a demonstrated analgesic effect and is also known to inhibit pruritus[4]. Moreover, intravenous nalbuphine has been shown to not only attenuate the incidence of pruritus but also to decrease total morphine consumption[5]. However, the mechanism underlying nalbuphine's mediation of morphine-induced scratching (MIS) is still not clearly understood.

Microglia are macrophages that reside in the central nervous system (CNS); they are known to play immunoregulatory roles within the CNS. Notably, they are involved in the regulation of both pain[6, 7] and itching. For example, recent research found that compound $48 / 80$ and 5 -guanidinonaltrindole (GNTI) elicited pronounced scratching behaviors in mice in conjunction with microglial activation in the mice's spinal cords[8]. Other research has also indicated the involvement of spinal microglia in both pruritogeninduced and chronic itching[9]. However, the precise role of microglia in MIS is still unclear, and it is 
unknown whether MIS is related to microglial activity or whether nalbuphine's inhibition of MIS is dependent on a microglial mechanism.

In the present study, we investigated i.t. injections of nalbuphine inhibited scratching behaviors in mice and examined the changes in microglial activation in the mice's spinal cords. We found that microglial activation mediated MIS and that nalbuphine inhibited MIS are by upregulating PKCD expression, while inhibited microglial activation and in turn affected the expression of phosphorylated-p38 (p-p38). Overall, our study defined the functional relationship between the KOR agonist nalbuphine and microglial activation in the modulation of MIS.

\section{Materials And Methods}

Animals Male C57BL/6J mice between 7 and 12 weeks of age were used for experiment.

They were purchased from the Guangdong Medical Laboratory Animal Center (GDMLAC).

All experiments were performed in accordance with the National Institutes of Health

Guideline and the International Association for the Study of Pain. Animal care procedures

were approved by the Animal Studies Committee at Guangzhou Medical University.

Animals were housed under a $12 \mathrm{~h}$ light/dark cycle in an area with free access to water and food.

Drugs and reagents Morphine were supplied from Northeast Pharmaceutical Group Shenyang First Pharmaceutical Co. Ltd. (Shenyang, China). Phosphorylate p38 were from Sigma (American). PKCa, PKC $\beta, P K C \delta$ siRNA were purchased from Thermo fisher (American) and in vivo-jetPEI® was from Polyplus-transfection (France).

\section{Itch models}

Acute itch of morphine-induced model The mice skin of back were shaved 3 days before $0.06 \mathrm{nmol}$ morphine was i.t injected after nalbuphine $(2 \mathrm{nmol})$ injection, the control group was i.t injected normal saline (NS), according to experiment design, after injection, each mice was return to the animal arena and videotape recording for 30 min immediately.

Itch behavioral Scratching behaviors were performed as previously described [10]. Behavioral tests were videotaped (HDR-CX190, Canon) at the animal plastic arena. The videos were played back on computer and the quantification of mice scratching behaviors were finished by observer who was blinded to the treatments. Hind paw scratching behaviors were recorded for 30 min with 5 min intervals. A battery of scratches were defined as a lifting of the hind limb to the painting (nape) or injection site and then a 
replacing of the limb back to the floor or to the mouth, regardless of how many scratching strokes take place in between[11].

Mechanical sensitivity test The mice of the two groups were tested for paw withdrawal mechanical threshold (PWMT) before and after given intrathecal (i.t.) injection of morphine. PWMTs were measured on day 0,1 and 3. The mechanical allodynia test method was described previously[12]. Mechanical sensitivity to mechanical stimulation was measured on a Dynamic Plantar Anesthesiometer apparatus (Ugo Basile, Comeria, Italy) according to the manufacturer's manual. Place the mice into the enclosure of Dynamic Plantar Anesthesiometer at least $30 \mathrm{~min}$ before the experiment to habituate them to the measurement conditions. The Dynamic Plantar Anesthesiometer pushed a thin rod (diameter $0.5 \mathrm{~mm}$ ) with increasing force through a wire-grated floor against the plantar surface of a hind paw from beneath. The force increased from 0 to $5 \mathrm{~g}$ within $10 \mathrm{~s}$ (ramp $0.5 \mathrm{~g} / \mathrm{s}$ ) and was then held at $5 \mathrm{~g}$ for an additional $10 \mathrm{~s}$. It stopped automatically and recorded the latency time when the animal withdraws the paw. Paw withdrawal mechanical threshold time was calculated as the mean of 6 consecutive stimulations of the hind paw at intervals of at least $10 \mathrm{~s}$.

Thermal hyperalgesia evaluation For the heat pain test, the mice were placed in the plate apparatus. The first ipsilateral hind paw flexion reflex was considered the nociceptive endpoint, which was recorded as paw withdrawal thermal latency (PWTL). The response latency was recorded before the morphine treatment and at the 1st and 3rd day post-administration morphine. The maximum latency (cut-off) was set at $20 \mathrm{~s}$ to avoid tissue damage. To obtain data purely derived from the treatment, the inhibition values are presented as the difference between the basal values of vehicle or drug-treated animals and the respective controls.

PKC siRNA treatment Before observe PKC isoform role in MIS or in nalbuphine treatment. The experiment group divided into three groups: Control group, treatment with saline plus scramble siRNA; morphine group, treatment with PKC siRNA first then i.t. morphine; Nalbuphine group, treatment with PKC siRNA first then i.t. nalbuphine plus morphine. PKCa, PKC $\beta$, PKC siRNA (Thermo Fisher) were delivered to the lumbar region of the spinal cord via i.t. injection as described [13]. In vivo jetPEI® as the transfection median was diluted by steriled DEPC.PBS $\cdot \mathrm{ddH}_{2} \mathrm{O}$. The three kinds of PKC siRNA were diluted with steriled DEPC.PBS. $d \mathrm{dd}_{2} \mathrm{O}$ in $0.5 \mathrm{ug} / \mathrm{ul}$ concentration, then mixed up the PEl solution with siRNA, and put into room temperature for $20 \mathrm{~min}$, total $2.5 \mu \mathrm{g} / 10 \mu \mathrm{l}$ mixture siRNA was i.t. injected into mice, the scramble siRNA was injected into another control group mice. Mice were injected twice a day for 5 consecutive days.

Immunohistochemistry Mice were deeply anaesthetized with 10\% chloralhydrate and perfused through right auricle with saline followed by $4 \%$ paraformaldehyde. After the perfusion, the spinal cords were removed and post-fixed in $4 \%$ paraformaldehyde overnight. Then, dehydration according to $10 \%, 20 \%$, $30 \%$ sucrose concentration gradient. Spinal cord tissues were cut into $20 \mu \mathrm{m}$ thickness. Free floating sections were incubated in blocking solution containing $2 \%$ donkey serum and $0.1 \%$ Triton X-100 in PBS (PBS-T) for $1 \mathrm{~h}$ at room temperature. Then incubated with primary antibodies overnight at $4{ }^{\circ} \mathrm{C}$, washed three times in PBS-T, incubated with the secondary antibodies for $2 \mathrm{~h}$ at room temperature and washed 
three times. Sections were mounted on slides with Fluoromount G (Southern Biotech) and coverslips. The following primary antibodies were used: Rabbit anti-Iba1 (1:400; Sigma), rabbit anti-KOR (1:200; Sigma), mouse anti-phosphorylate-p38(1:200; Cell Signaling), mouse anti-lba1 (1:400, Thermo Fisher). The secondary antibodies were purchased from Jackson Immuno Research Laboratories including Dylight 488 goat anti-rabbit antiserum $(1: 500,1.25 \mu \mathrm{g} / \mathrm{ml})$ and Dylight 594 goat anti-mouse $(0.33 \mu \mathrm{g} / \mathrm{ml})$. Images were taken using a Nikon Eclipse Ti-U microscope.

Western Blot Briefly, tissues were homogenized at $4{ }^{\circ} \mathrm{C}$ with an electric homogenizer (COYOTE, China) in RIPA with complete protease inhibitor cocktail (Selleck, USA). The homogenate was centrifuged at 12000 $\times \mathrm{g}$ for $5 \mathrm{~min}$ at $4{ }^{\circ} \mathrm{C}$ and retained the supernatant. Protein concentration was determined using a MicroBCA protein assay kit (Pierce, cat. 23235, Rockford, IL, USA) and BCA test method. Protein samples were diluted with the $2 \times$ electrophoresis sample buffer, boiled for $5 \mathrm{~min}$ and rapid cooling on ice. For western blotting, proteins were electrophoretically transferred from resolving gels to nitrocellulose membranes $(0.2 \mu \mathrm{m}$, Bio-Rad) in transfer buffer [192 mM glycine, $25 \mathrm{mM}$ Tris $\cdot \mathrm{HCl}(\mathrm{pH} 8.3)$ and $20 \%$ methanol] for $1 \mathrm{~h}$ at $100 \mathrm{~V}$ (Bio-Rad). Blotted membranes were washed twice with TBS-T(pH 7.4), and blocked for $1 \mathrm{~h}$ at room temperature with the mixture of milk and TBS-T. Blots were incubated with $C D 11 \mathrm{~b}(1 \mathrm{ug} / \mathrm{ml}$, Sigma), Phosphorylated-p38(1:5000, CST), PKCa, PKC, PKCס(1:5000, Santa Cruz) antibodies overnight at $4{ }^{\circ} \mathrm{C}$ plus $1 \mathrm{~h}$ at room temperature. After washing twice with TBS-T (pH 7.4), the membrane was incubated with secondary antibodies HRP Goat anti-Mouse (Proteintech, China, dil 1:5000) and HRP Goat anti Rabbit (Proteintech, China, dil 1:5000) in TBST for $1 \mathrm{~h}$ at room temperature. Then visualized with the GenoSens 1510 Gel Imaging System (Clinx, China).

Quantification and statistics Statistical comparisons were performed with GraphPad Prism 7. All data are presented as means \pm SEM. Student $t$ test was used for inter-group comparison, and one way-ANOVA was used for intra-group comparison. $P$ values $<0.05$ were considered to be significant.

\section{Results}

\section{Nalbuphine significantly inhibited MIS but had no effect on mechanical and thermal pain behaviors}

MIS has been previously used to study itching[14], and KOR agonists, including nalbuphine, have been previously used to suppress itch, including MIS[15]. In the present study, we created an acute MIS model to more precisely determine the relevant effects of nalbuphine and its underlying mechanisms. Consistent with previous research[16], we found that nalbuphine significantly reduce MIS behaviors in 10-15 min after intrathecal injections when recording $30 \mathrm{~min}$ (Fig. 1A). Meanwhile, we observed that nalbuphine's inhibition of MIS was dose-dependent, with maximum inhibition observed at a dose of $2 \mathrm{nmol}$ (Fig. 1B). We thus confirmed that nalbuphine dose-dependent inhibitory effect on MIS. However, the withdrawal thresholds for both mechanical and thermal pain were not significantly different between the morphine i.t. and nalbuphine treatment groups (Fig. 1C, 1D). 


\section{Microglial activation was involved in MIS and Iba1 was co-expressed with KOR}

Although previous research has observed that microglia mediate itch sensations[8], there has been a lack of research regarding microglial involvement in MIS. In order to address this research gap, we gave i.t. injections of morphine to mice and then used immunohistochemical and western blot analyses to observe the mice's microglial expressions during their immediate MIS responses. We found that ionized calcium-binding adapter molecule-1 (Iba1), which is a marker of microglial, was significantly increased in the spinal cord laminae I-V of mice in the treatment group; in the control group, lba1 was only present in laminae I and II (Fig. 2C, 2D). Morphologically, the microglial cell bodies were hypertrophic, with thickened and retracted processes, and their cell numbers were higher than in control mice. Increased CD11b, another marker of microglial, was also observed in mice exhibiting MIS (Fig. 2A, 2B). Furthermore, after our administration of $2 \mathrm{nmol}$ nalbuphine, the expression of Iba1 decreased significantly and even similarly closed located in laminae I and II (Fig. 2C). Western blot analyses also showed decreased CD11b expression in the nalbuphine treatment group.

We therefore next sought to investigate whether KORs activation inhibited microglial activation directly or indirectly. Using immunofluorescence, we found that KORs and Iba1 were co-expressed in the mice's spinal cords after nalbuphine treatment, as expected (Fig. 2C)[17]. More specifically, KOR expression increased 20 min post-injection of nalbuphine, following a reduction of activated microglia. Moreover, although the total number of microglia decreased, most of the remaining microglia were co-expressed with KORs. These results suggest that nalbuphine may activate KORs, which in turn inhibit microglial activation, thereby diminishing MIS behaviors.

\section{The antipruritic effects of nalbuphine were PKCס-dependent and MIS was PKC $\beta$-dependent}

Previous research has shown that PKCs mediate both KOR activation and a non-canonical opioid signaling mechanism in which Gastrin releasing peptide receptor (GRPR) activity is attenuated by KORmediated cross-signaling in mouse spinal cords[13,18]. These results led us to investigate which PKC isoform mediated KOR activation and inhibited itching. We thus performed three PKC knockdown studies in which mice were treated with either PKCa, PKC $\beta$, or PKCס siRNA[19].

We found that the PKC $\delta$ isoform was involved in KOR-related itching inhibitions: when we performed i.t. injections of PKC $\delta$ siRNA twice a day for 3 days, the PKC $\delta$ siRNA knockdown blocked nalbuphine's inhibition of MIS, as compared with the other siRNA treatment mice (Fig. 3A). However, PKCס knockdowns had no effect on mice that received only morphine (Fig. 3B). Similarly, we found that the expression of PKC $\delta$ decreased after an initial i.t. morphine treatment, as compared with C57/BL6 control mice, but increased after subsequent treatment with nalbuphine (Fig. 3E), suggesting that nalbuphine's observed antipruritic effect was PKC $\delta$-dependent.

We next tested the effects of PKC $\beta$ siRNA on MIS and found that knockdown PKC $\beta$ mice had attenuated MIS; they scratched significantly less than scramble siRNA control mice (Fig. 3C). However, nalbuphine's effects were the same in the scramble siRNA control mice and PKC $\beta$ knockdown mice (Fig. 3D). Similarly, 
western blot analyses showed that PKC $\beta$ were significantly increased in the morphine-treated mice and, accordingly, decreased after injection with nalbuphine (Fig. 3F). These results suggest that MIS is PKC $\beta-$ dependent.

\section{Pkc May Affect Microglial Activation And Induce Kor Activation}

To further evaluate the role of PKCs in MIS and in the KOR activation-induced inhibition of microglia, we first examined the PKC isoform that influenced microglial activation. Notably, we found that the microglia were activated after PKC $\delta$ siRNA treatment in our MIS model and that CD11b protein levels (a marker of microglial activation) were increased in both the morphine group and, especially, in the morphine plus nalbuphine group, as compared to the scramble siRNA control group (Fig. 4A). This is consistent with our previous results that the activation of microglia attenuated after nalbuphine treatment (Figs. 2A, 2B and 2C) but that, in knockdown PKC $\delta$ mice, CD11b increased more significantly after nalbuphine treatment for MIS (Fig. 4A). Furthermore, CD11b expression decreased in the morphine group after PKC $\beta$ siRNA treatment, thus confirming again that the morphine-induced microglial activation was PKC $\beta$-dependent.

However, since microglial levels did not change significantly after treatment with nalbuphine in PKC $\beta$ siRNA mice (Fig. 4B), they may also be dependent on an additional underlying mechanism. Therefore, we next investigated whether the microglial activation marker Iba1 was co-expressed with either PKC $\beta$ or PKC $\delta$ in the morphine and nalbuphine treatment mice. We found that Iba 1 expression was correlated with the expressions of both PKC $\delta$ and PKC $\beta$ (Figs. 4C, 4D); however, in the nalbuphine-treated MIS group, PKCठ expression increased while Iba1 expression decreased (Figs. 4C). This abnormal expression relationship thus indirectly confirmed that KORs may activate PKC $\delta$ but cannot trigger sufficient microglial activation, thereafter, resulting in the inhibition of MIS. Moreover, we also found that PKC $\beta$ expression increased in laminae I and II after MIS was triggered, but decreased significantly after subsequent nalbuphine treatment (Fig. 4D). These results are consistent with our previously described western blot results (Fig. 3F).

The p38 mitogen-activated protein kinase (MAPK) microglial pathway was inhibited after KOR activation

Microglial activation after MIS led us to hypothesize that the p38 MAPK pathway was inhibited after nalbuphine-induced KOR activation. To investigate the involvement of the p38 MAPK pathway, we immunostained spinal cord sections for p-p38 expression after nalbuphine treatment. We found that pp38 expression increased significantly in the morphine-treated mice as compared to the control mice; however, the positive p-p38 immunostained cells decreased after treatment with nalbuphine. Iba1 was also co-expressed with p-p38, and their expressions were consistent with each other in the spinal cord (Fig. 5B). We also examined p-p38 expression using western blot analyses and found that p-p38 protein levels in the spinal cord were increased in the morphine-treated mice but decreased after subsequent 
treatments of nalbuphine (Fig. 5A). Taken together, these results suggest that p-p38 expression levels in the activated microglia were involved in both MIS and in the KOR-mediated inhibition of itching.

\section{Discussion}

Itching is a pathological process in the somatosensory system at the levels of the primary sensory neurons, spinal cord, and brain. Although there has been distinct progress in the itching research field, the mechanisms underlying the sensation and transmission of itching, and in particular morphine-induced itch, are still unclear. Research over the past decade has increasingly shown that pain and itch are two clearly distinct sensations[20], and recent studies have identified separate neuronal pathways involved in each sensation[21].

One proposed mechanism for chronic itch involves an abnormal excitability of pruritic-specific neurons, such as the GRPR and NPPA neurons in the spinal dorsal horn[13, 22, 23]. However, although GRPR has been shown to be involved in MIS[19], an increasing body of evidence suggests that synaptic hyperexcitability in the spinal dorsal horn may not be a consequence simply of neuronal changes but may also be involved in multiple glial cell alterations[6].

The important role of microglia in itching sensations has also been verified in a series of studies[8, 2426]. As CNS-dwelling macrophages, it is known that microglia play an important immunoregulatory role within the CNS [27]. Moreover, it has been shown that microglia become activated in the spinal dorsal horn after peripheral nerve damage [8]. Previous studies have also revealed the involvement of spinal microglia in pruritogen-induced itching, and recent research has shown that compound 48/80 and GNTI can elicit pronounced scratching behaviors in mice in conjunction with microglial activation in mouse spinal cords[21]. However, it remains rather unclear exactly how microglia mediate MIS and how they affect the KOR activation process.

Previous evidence has suggested that PKC $\delta$ could play a specific role as an intracellular modulator of itch in sensory neurons[28]. It is also known that KOR activation attenuates itch via PKCס, although the broader role of PKCs in the development of acute itch has not yet been elucidated. We therefore set out to investigate PKC mechanisms, and we found that PKC $\delta$ affected the KOR-mediated inhibition of MIS. However, although PKC $\delta$ could still be activated by the administration of nalbuphine in mice treated with PKC $\delta$ siRNA, it had less effect on MIS. These observations are consistent with the hypothesis that PKC $\delta$ is activated in the KOR-mediated inhibition of MIS[13].

In addition, we also found that CD11b levels demonstrated an opposite trend to that of PKC $\delta$. Since microglia were activated after treatment with PKC $\delta$ siRNA in the nalbuphine plus morphine group, it is clear that PKC had a negative feedback regulation of morphine, and this is also consistent with previous research. In contrast, mice treated with $\mathrm{PKC} \beta$ siRNA demonstrated that $\mathrm{PKC} \beta$ positively regulated microglia. The MIS model was therefore associated with increased PKC $\beta$ expression, which was in turn inhibited by nalbuphine. The expressions of both PKC $\delta$ and PKC $\beta$ were also both associated with microglial activation. However, whether PKC $\delta$ activates microglia directly via phosphorylation or indirectly 
via other kinases requires additional research[29]. We were also unable to identify any role for PKCa in acute itching.

Our research used knockdown PKC isoforms to observe the role of microglia in MIS and to explore the relationship between microglial activation and the KOR/PKC pathways in nalbuphine's antipruritic effect. Using a series of pharmacological, behavioral, immunochemical, and molecular biology experiments, we first found that the KOR agonist nalbuphine inhibited MIS in a dose-dependent manner and that this inhibitory effect was PKCס-dependent. Second, we demonstrated that MIS could induce microglial activation and that this activation was PKC $\beta$ dependent. Third and finally, we found that nalbuphine inhibited MIS via the retroegulation of microglial activation by the KOR-PKC $\delta$ pathway. Our research has thus identified a new mechanism underlying nalbuphine's antipruritic effect and provides novel insights about MIS that may lead to drug targets.

Moreover, previous research has shown that KOR agonists are effective at suppressing scratching in mice treated with different pruritogens[30]. Nalbuphine is a nonscheduled KOR agonist and mu opioid receptor antagonist that has been approved by the FDA since the 1980s for clinical treatment of moderate to severe pain, including in postoperative pain management; more recently, it has also been studied in clinical trials as a treatment for chronic itch, or prurigo- nodularis. Although the use of nalbuphine as a treatment for the pruritus associated with neuraxial opioid use has not been approved by the FDA, it has nevertheless been used in this capacity. In fact, one study found that nalbuphine was a better treatment for MIS than either a placebo, a control, diphenhydramine, naloxone, or propofol in patients receiving neuraxial opioids for acute pain related to surgery or childbirth [31]. However, the mechanism underlying nalbuphine's antipruritic effects is still unclear.

There has been relatively little research on this topic to demonstrated that spinal KOR activation reduced itch transmission by inhibiting the function of GRPR. However, this is the only study to date that has investigated the role of a KOR agonist in MIS, and it investigated the role of neurons rather than microglia. The present research demonstrated that microglia's immunoregulatory roles may be involved in MIS and that nalbuphine may affect microglial functioning in some manner. As expected, we observed significantly increased microglial activation after morphine i.t. injections; moreover, this activation was related to PKC $\beta$ function. Since this is the first time that this phenomenon has been observed in MIS, we used siRNA knockdowns to confirm that it was PKC isoform-specific; we observed that, in knockdown PKC $\beta$ mice, microglial activation was dramatically inhibited following decreases in MIS, thus demonstrating PKC $\beta^{\prime}$ s importance in MIS.

Two recent photon in vivo imaging studies found that microglial processes are highly dynamic in the brain and spinal cord[32]: stimulated microglia rapidly move toward the site of an injury and directly appose synaptic regions, in response to neuronal activity, thereafter facilitating contact with highly active neurons[25]. Microglial activation had not been previously reported in MIS[33]. Moreover, KOR activation was thought to attenuate the expression of microglia via some other mechanism than the G-protein signaling pathway[34]. However, we found that microglia were activated in the spinal dorsal horns. 
Microglia are known to dramatically alter gene expressions at a molecular level, including via Iba1, CD11, and p-p38, and our results demonstrate that microglia play an important role in physical condition monitoring, such as itching, regardless of the severity of an injury[35].

Research has also shown that various microglia signaling molecules, including cell-surface receptors, are increased in the spinal dorsal horn once microglia are activated following a nerve injury[36]. Following a nerve injury, the phosphorylation of p38 MAPK increases, although this is highly restricted to spinal microglia[37]. In vitro studies have furthermore shown that activated p38 MAPK is present in spinal microglia and participates in the signaling pathways that mediate nociception at the spinal level [38]. Microglia and p-p38 have also been shown to have a role in chronic itch[9].

Since we knew the potential roles of PKCs and microglia, we therefore hypothesized that p38 MAPK was also involved[39]. An examination of p-p38 expression showed increased levels of p-p38 after treatment with morphine that were furthermore inhibited by nalbuphine. Iba1 cells were also co-expressed with pp38 in the spinal cord, confirming our western blot results. These results suggest that microglial activation may occur by phosphorylating p38 to maintain an acute MIS signal. Our previous research has also suggested that the microglial inhibitor minocycline inhibited the p-p38 MAPK pathway in our MIS model[40]. Overall, our present results suggest that, as the regular downstream microglial pathway, p38 MAPK precisely regulated microglia during the KOR-mediated inhibition of MIS. Although significant prior evidence existed that microglia play a role in chronic itch and pain, the field has lacked a deep understanding of microglia's role in pruritus-induced itching and in neuraxial opioid-induced itching. Although our research observed an increased expression of microglia and the involvement of the p-p38 pathway, the exact microglial mechanisms underlying MIS still require further research.

Taken together, our acute itch mouse experiments have suggested a new regulation mechanism involving KORs and microglia. Moreover, the signaling pathway that we identified here as a mediator of acute MIS is similar to the one involved in pain, which also hinges on microglia and p-p38 activation. Ultimately, our findings about PKC isoform involvement and the downstream effects of p38 MAPK and KOR activation suggest new ways to treat MIS.

\section{Declarations}

\section{Ethics approval and consent to participate}

The study was approved by the Animal Studies Committee at Guangzhou Medical University, China.

\section{Consent for publication}

Not applicable.

\section{Availability of data and materials}


The datasets generated and/or analyzed in this study are available from the author on reasonable request.

\section{Funding}

The research was supported by the National Natural Science Foundation of China (No. 81771182) to L Wan, by Natural Science Foundation of Guangdong Province (No.2016A030313599) to L Wan.

\section{Competing interests}

The authors report no conflicts of interest in this work.

\section{Authors' contributions}

LW designed the experiments, YT, NL and SH performed behavioral, immunohistochemistry and molecular biology experiments. YT performed molecular analysis, ZP contributed to the project, YT and LW wrote the manuscript and LW revised the writing. All authors read and approved the final manuscript.

\section{Acknowledgements}

Not applicable.

\section{References}

1. Han L, Dong X. Itch Mechanisms and Circuits. Annual Review of Biophysics. 2014; 1(43).

2. -355 .

3. Tan X, Shen L, Wang L, Zhang Y, Zhang X, Huang Y. Incidence and risk factors for epidural morphine induced pruritus in parturients receiving cesarean section: A prospective multicenter observational study. Medicine. 2019;40(98):98:40.

4. Benson JL, Campbell HE, Phillips CN. Opioid-induced pruritus. Consult Pharm. 2015;4(30):221-7.

5. Narver HL. Nalbuphine, a non-controlled opioid analgesic, and its potential use in research mice. Lab Anim (NY). 2015;3(44):106-10.

6. Chen MK, Chau SW, Shen YC, Sun YN, Tseng KY, Long CY, Feng YT, Cheng KI. Dose-dependent attenuation of intravenous nalbuphine on epidural morphine-induced pruritus and analgesia after cesarean delivery. Kaohsiung J Med Sci. 2014;5(30):248-53.

7. Calvo M, Bennett DLH. The mechanisms of microgliosis and pain following peripheral nerve injury. Exp Neurol. 2012;2(234):271-82.

8. Mika J, Zychowska M, Popiolek-Barczyk K, Rojewska E, Przewlocka B. Importance of glial activation in neuropathic pain. Eur J Pharmacol. 2013; 1-3(716); 106 - 19.

9. Zhang Y, Dun SL, Chen Y, Luo JJ. A. Cowan and N.J. Dun. Scratching activates microglia in the mouse spinal cord. J Neurosci Res. 2015;3(93):466-74. 
10. Zhang Y, Yan J, Hu R, Sun Y, Ma Y, Chen Z, Jiang H. Microglia are Involved in Pruritus Induced by DNFB via the CX3CR1/p38 MAPK Pathway. Cell Physiol Biochem. 2015;3(35):1023-33.

11. Sun YG, Zhao ZQ, Meng XL, Yin J, Liu XY, Chen ZF. Cellular basis of itch sensation. Science. 2009;5947(325):1531-4.

12. Sun YG, Chen ZF. A gastrin-releasing peptide receptor mediates the itch sensation in the spinal cord. Nature. 2007;7154(448):700-3.

13. Russe OQ, Möser CV, Kynast KL, King TS, Stephan H, Geisslinger G, Niederberger E. Activation of the AMP-Activated Protein Kinase Reduces Inflammatory Nociception. The Journal of Pain. 2013;11(14):1330-40.

14. Munanairi A, Liu XY, Barry DM, Yang Q, Yin JB, Jin H, Li H, Meng QT, Peng JH, Wu ZY, Yin J, Zhou XY, Wan L, Mo P, Kim S, Huo FQ, Jeffry J, Li YQ, Bardoni R, Bruchas MR, Chen ZF. Non-canonical Opioid Signaling Inhibits Itch Transmission in the Spinal Cord of Mice. 2018. p. 866-877.

15. Miyamoto T, Patapoutian A. Why Does Morphine Make You Itch? Cell. 2011;2(147):261-2.

16. Brust TF, Morgenweck J, Kim SA, Rose JH, Locke JL, Schmid CL, Zhou L, Stahl EL, Cameron MD, Scarry SM, Aube J, Jones SR, Martin TJ, Bohn LM. Biased agonists of the kappa opioid receptor suppress pain and itch without causing sedation or dysphoria. Sci Signal. 2016;456(9):ra117.

17. Sakakihara M, Imamachi N, Saito Y. Effects of Intrathecal kappa-Opioid Receptor Agonist on Morphine-Induced Itch and Antinociception in Mice. Reg Anesth Pain Med. 2016;1(41):69-74.

18. Ito D, Imai Y, Ohsawa K, Nakajima K, Fukuuchi Y, Kohsaka S. Microglia-specific localisation of a novel calcium binding protein, Iba1. Brain Res Mol Brain Res. 1998;1(57):1-9.

19. Ally RA, Ives KL, Traube E, Eltounsi I, Chen P, Cahill PJ, Battey JF, Hellmich MR, Kroog GS. Agonistand Protein Kinase C-Induced Phosphorylation Have Similar Functional Consequences for GastrinReleasing Peptide Receptor Signaling via Gq. Mol Pharmacol. 2003;4(64):890-904.

20. Liu X, Liu Z, Sun Y, Ross M, Kim S, Tsai F, Li Q, Jeffry J, Kim J, Loh HH. and Z. Chen. Unidirectional Cross-Activation of GRPR by MOR1D Uncouples Itch and Analgesia Induced by Opioids. Cell. 2011;2(147):447-58.

21. Liu T, Ji R. New insights into the mechanisms of itch: are pain and itch controlled by distinct mechanisms? Pflügers Archiv -. European Journal of Physiology. 2013;12(465):1671-85.

22. Luo J, Feng J, Liu S, Walters ET, Hu H. Molecular and cellular mechanisms that initiate pain and itch. Cell Mol Life Sci. 2015;17(72):3201-23.

23. Zhao ZQ, Wan L, Liu XY, Huo FQ, Li H, Barry DM, Krieger S, Kim S, Liu ZC, Xu J, Rogers BE, Li YQ, Chen ZF. Cross-Inhibition of NMBR and GRPR Signaling Maintains Normal Histaminergic Itch Transmission. J Neurosci. 2014;37(34):12402-14.

24. Tsuda M. Modulation of Pain and Itch by Spinal Glia. Neurosci Bull. 2018;1(34):178-85.

25. Mika J. Modulation of microglia can attenuate neuropathic pain symptoms and enhance morphine effectiveness. Pharmacol Rep. 2008;3(60):297. 
26. Peng J, Gu N, Zhou L, Eyo UB, Murugan M, Gan W, Wu L. Microglia and monocytes synergistically promote the transition from acute to chronic pain after nerve injury. Nature Communications. 2016; 7); 12029.

27. Mika J. Modulation of microglia can attenuate neuropathic pain symptoms and enhance morphine effectiveness. Pharmacol Rep. 2008;3(60):297-307.

28. Tsuda M. Microglia in the CNS and Neuropathic Pain. Adv Exp Med Biol. 2018;11(1099):77-91.

29. Valtcheva MV, Davidson S, Zhao C, Leitges M. and R.W. Gereau. Protein Kinase C $\delta$ Mediates Histamine-Evoked Itch and Responses in Pruriceptors. Molecular Pain. 2015; 11); 1744-8069-11-1.

30. Kelly E, Bailey CP, Henderson G. Agonist-selective mechanisms of GPCR desensitization. Br J Pharmacol. 2008;153(Suppl 1):379-88.

31. Dunn AD, Reed B, Guariglia C, Dunn AM, Hillman JM. and M.J. Kreek. Structurally Related Kappa Opioid Receptor Agonists with Substantial Differential Signaling Bias: Neuroendocrine and Behavioral Effects in C57BL6 Mice. Int J Neuropsychopharmacol. 2018;9(21):847-57.

32. Jannuzzi RG. Nalbuphine for Treatment of Opioid-induced Pruritus: A Systematic Review of Literature. The Clinical Journal of Pain. 2016;1(32):87-93.

33. Davalos D. and K. Akassoglou. In vivo Imaging of the Mouse Spinal Cord Using Two-photon Microscopy. Journal of Visualized Experiments. 2012; 59.

34. Woods JM, Lim AG. Prevalence and management of intrathecal morphine-induced pruritus in New Zealand Māori healthcare recipients. British Journal of Pain. 2017;1(12):20-5.

35. Schattauer SS, Kuhar JR, Song A, Chavkin C. Nalfurafine is a G-protein biased agonist having significantly greater bias at the human than rodent form of the kappa opioid receptor. Cell Signal. 2017;32:59-65.

36. Tsuda M, Mizokoshi A, Shigemoto Mogami Y, Koizumi S, Inoue K. Activation of p38 mitogenactivated protein kinase in spinal hyperactive microglia contributes to pain hypersensitivity following peripheral nerve injury. Glia. 2004;1(45):89-95.

37. Ji R. and M.R. Suter. p38 MAPK, Microglial Signaling, and Neuropathic Pain. Molecular Pain. 2007; 3); 1744-8069-3-33.

38. Obata T, Brown GE, Yaffe MB. MAP kinase pathways activated by stress: the p38 MAPK pathway. Crit Care Med. 2000;4(28):N67-77.

39. Svensson $\mathrm{Cl}$, Marsala M, Westerlund A, Calcutt NA, Campana WM, Freshwater JD, Catalano R, Feng Y, Protter AA, Scott B, Yaksh TL. Activation of p38 mitogen-activated protein kinase in spinal microglia is a critical link in inflammation-induced spinal pain processing. J Neurochem. 2003;6(86):1534-44.

40. Tsuda M, Inoue K, Salter MW. Neuropathic pain and spinal microglia: a big problem from molecules in "small" glia. Trends Neurosci. 2005;2(28):101-7.

41. Tang Y, Li N, Peng Z, Huang S, Li W. Activation of microglia in spinal cord is involved in morphineinduced itch. Chin J Pain Med. 2020;26(12):911-7. 


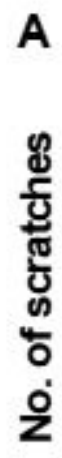

C

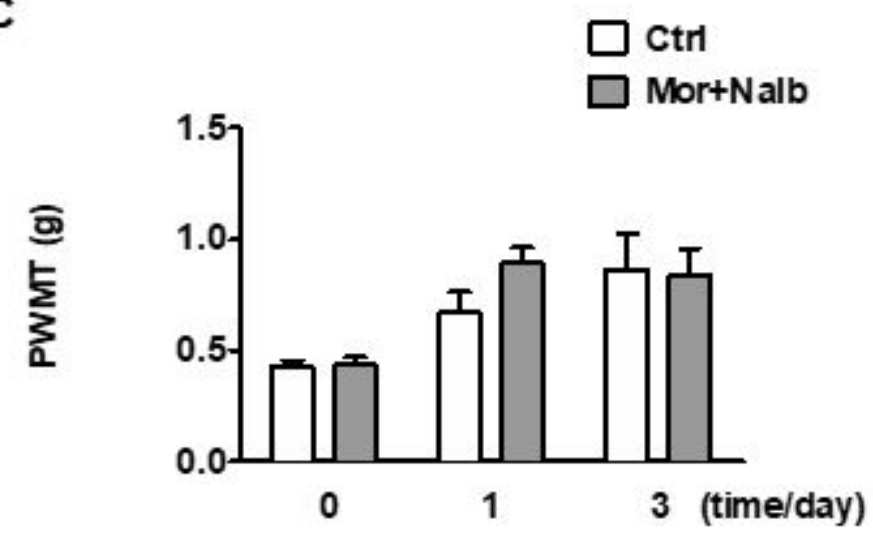

B

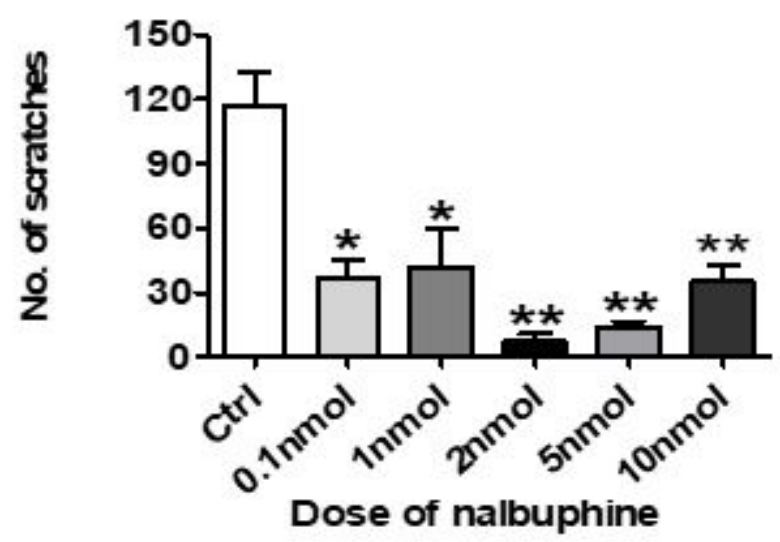

D

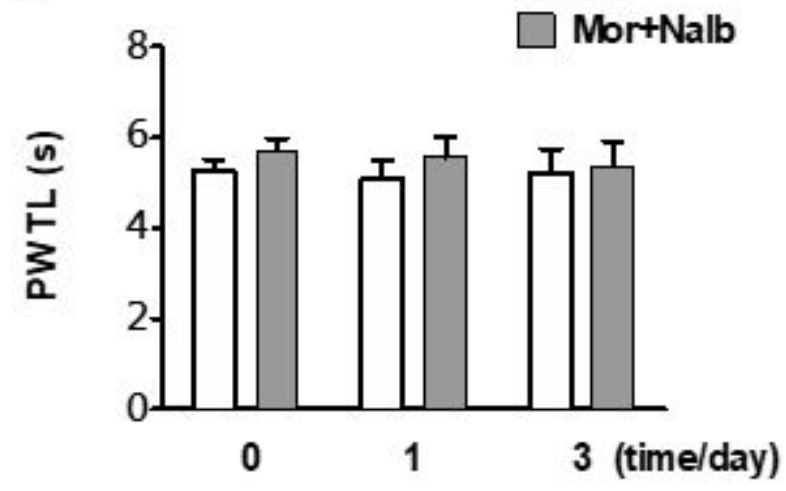

Figure 1

Intrathecal administration of nalbuphine can attenuate morphine induced scratch, but have no effect on mechanical and thermal pain. (A) 15 min pre-injection of nalbuphine (1nmol) significantly inhibit scratching induced by morphine $(0.6 \mathrm{nmol}$ i.t) when compared to their wide-type littermate which only i.t. morphine. ${ }^{*}<0.05$; Student unpaired $t$ test; $n=6$ for each group. (B) Different doses of nalbuphine $(0.1 \mathrm{nmol}, 1 \mathrm{nmol}, 2 \mathrm{nmol}, 5 \mathrm{nmol}, 10 \mathrm{nmol})$ attenuate pruritus induced by morphine to varying degrees when compared with i.t. morphine alone. ${ }^{*} P<0.05 ;{ }^{*} P<0.001$; Student unpaired $t$ test, $n=6$ for each group. (C) Mechanical withdrawal threshold was similar between treatment mice and C57 control mice. $P>0.05, n=6$. (D) Hind paw withdrawal latency was comparable between morphine acute itch model mice and their C57 control mice as tested by Hargreaves apparatus. $P>0.05, n=6$. 

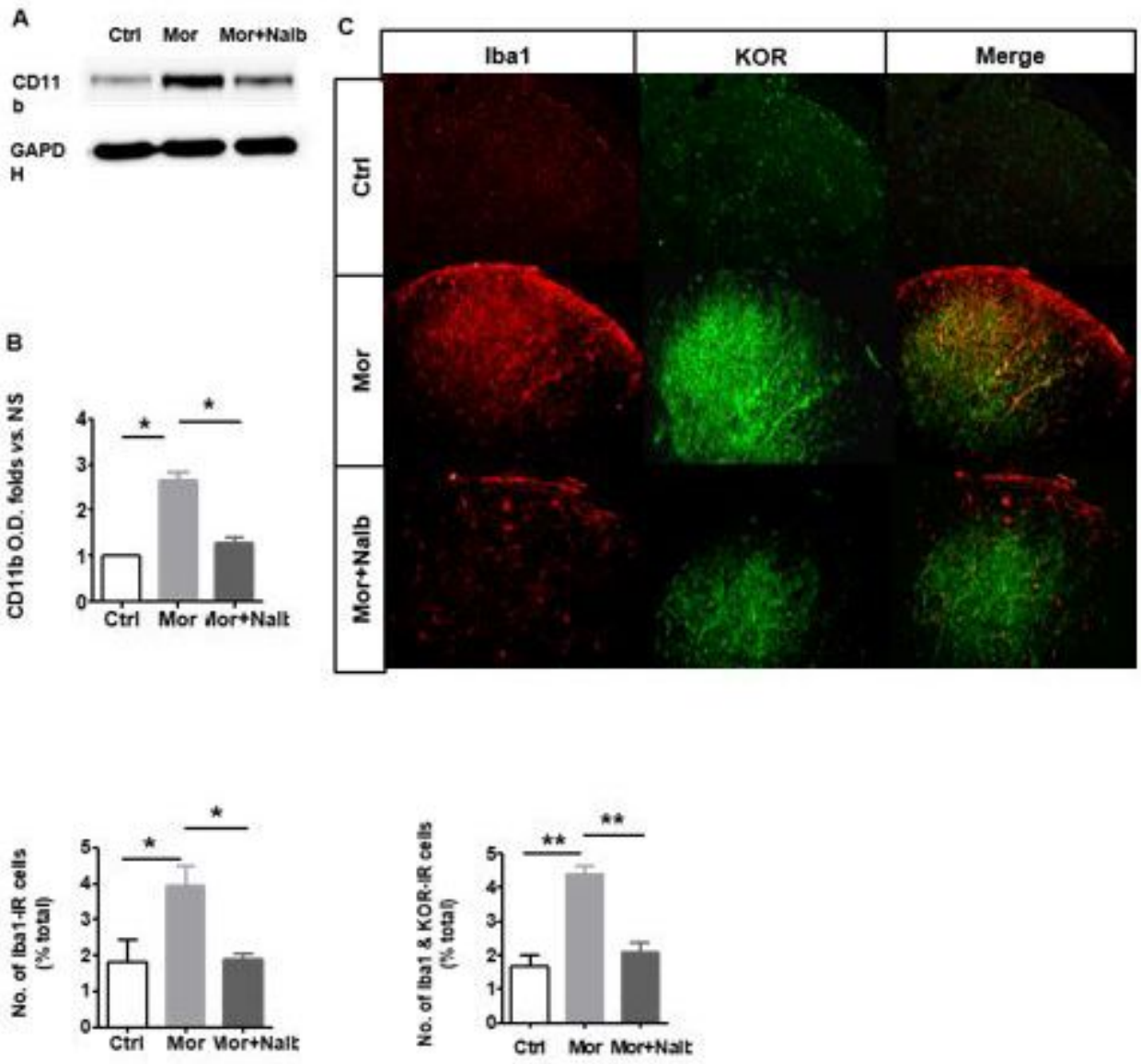

\section{Figure 2}

Microglia activation in the spinal cord were attenuated after nalbuphine treatment. (A) Representative western blot of CD11b expression in spinal cord extracts from control and morphine-treated mice; additional animal received intrathecal nalbuphine was analyzed at the same time with morphine-treated group mice. (B) Bar graph showing the quantity of CD11b through western blotting per spinal cord from animals with treatments indicated in (A). After morphine i.t. administration, the expression of microglia CD11b increased significantly when compared to control group, but significantly decreased after nalbuphine treatment. * $P<0.05$; bars represent mean $\pm S E M ; n=6$ for each group. (C) Representative images of lumbar spinal cord sections immunostained with anti-lba1 and anti-KOR after morphine treatment; additional animals received concomitant treatment with nalbuphine and were analyzed after these treatments. (D) The number of Iba1+ neurons and overlay with KOR (green) in the spinal cord of nalbuphine treatment model mice were significantly decreased comparing to morphine administrated mice; $n=6,{ }^{*} P<0.05,{ }^{\star *} P<0.01$, unpaired t test. 

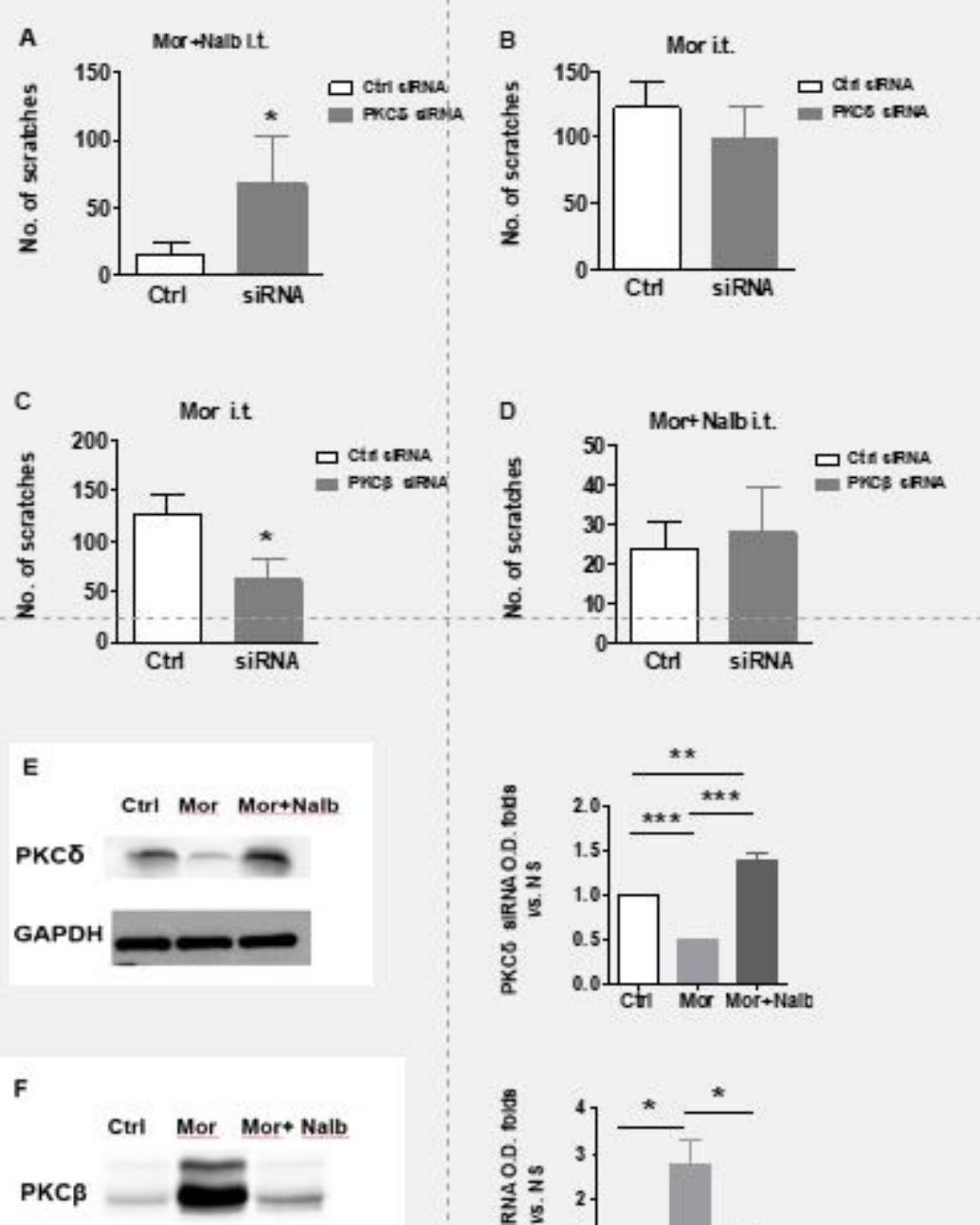

GAPDH

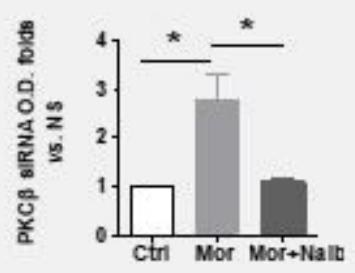

\section{Figure 3}

Nalbuphine attenuate MIS via decreased PKC $\beta$ and increased PKC $\delta$. (A) Bar graph showing the number of hind paw scratches in $30 \mathrm{~min}$ from animals receiving morphine $(0.06 \mathrm{nmol})$ after intrathecal with nalbuphine ( $2 \mathrm{nmol}$ ) between the PKCठ siRNA knockdown mice and scramble siRNA control mice. The nalbuphine inhibited MIS can be reversed by PKC $\delta$ siRNA knockdown. ${ }^{*} \mathrm{P}<0.05$; bars represent mean \pm SEM; $n=6$ for each group. (B) Bar graph showing the comparable number of hind paw scratches in 30 min from animals receiving morphine $(0.06 \mathrm{nmol})$ alone between the siRNA PKC $\delta$ knockdown mice and scramble siRNA control mice. $P>0.05$; bars represent mean $\pm S E M ; n=6$ for each group. (C) Graph showing the number of hind paw scratches in $30 \mathrm{~min}$ from animals receiving morphine $(0.06 \mathrm{nmol})$ alone between the siRNA PKC $\beta$ knockdown mice and scramble siRNA control mice. The MIS can be inhibited by PKC $\beta$ siRNA knockdown. * $P<0.05$; bars represent mean $\pm S E M ; n=6$ for each group. (D) The graph showing that there was no difference between control mice and PKC $\beta$ siRNA-treated mice after intrathecal injection with morphine and nalbuphine. $P>0.05$; bars represent mean $\pm S E M ; n=6$ for each group. (E) Western blot and quantified data showed the protein level of PKC $\delta$ was decreased after 
treatment with morphine while it was increased after nalbuphine treatment. ${ }^{* * P}<0.01,{ }^{\star \star *} P<0.001$; bars represent mean $\pm S E M ; n=6$ for each group. $(F)$ In contrast, western blot and quantified data showing that PKC $\beta$ expressed more after i.t injection morphine than the wild-type littermates, while the express of it decreased after treatment with nalbuphine. ${ }^{*} P<0.05$; bars represent mean $\pm S E M ; n=6$ for each group.

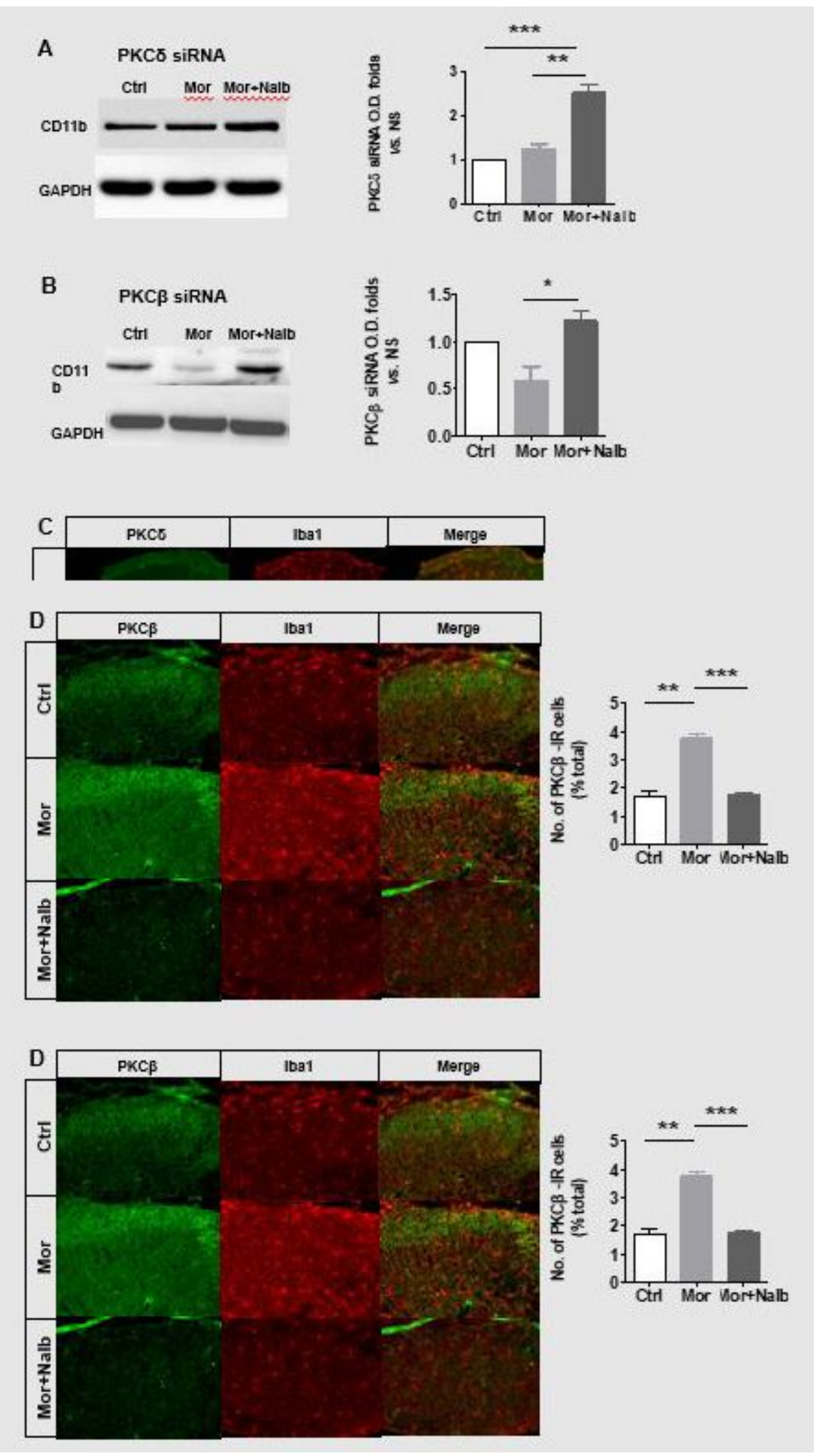

Figure 4 
PKC $\delta$ and PKC $\beta$ are influenced in the express of CD11b. (A) Western blot and quantified data showed the protein level of $C D 11 \mathrm{~b}$ was slightly increased after treat the PKC $\delta$ siRNA mice with morphine while it was significantly increased after nalbuphine treatment in contrast. ${ }^{\star *} \mathrm{P}<0.01,{ }^{\star} * \star \mathrm{P}<0.001$; bars represent mean \pm SEM; $n=6$ for each group. (B) Western blot and quantified data showed the protein level of CD11b decreased after PKC $\beta$ siRNA treatment in morphine model mice while had more effect on nalbuphinetreated mice in contrast. ${ }^{*} P<0.05$; bars represent mean $\pm S E M ; n=6$ for each group. (C) Representative images of spinal cord sections immunostained with anti-Iba1 and anti-PKC $\delta$ after morphine treatment; additional animals received concomitant treatment with nalbuphine following morphine treatment. Bar graph showing the number of PKC immunoreactive (IR) cells per spinal cord section from animals with treatments indicated before and the PKC $\delta-I R+$ and Iba1-IR+ cells were expressed more in nalbuphine treatment mice than morphine alone treated mice. ${ }^{*} \mathrm{P}<0.01$; bars represent mean $\pm S E M ; n=6$ for each group; Six sections per animal were selected for counting. (D) Representative images of spinal cord sections immunostained with anti-lba1 and anti-PKC $\beta$ after morphine treatment; additional animals received concomitant treatment with nalbuphine following morphine treatment. Bar graph showing the number of $P K C \beta$ immunoreactive (IR) cells per spinal cord section from animals with treatments indicated before and the PKC $\beta-\mathrm{IR}+$ cells were expressed more in morphine treatment mice than combine nalbuphine treated mice, but the Iba1-IR+ expression was more in nalbuphine treated mice than morphine mice. ${ }^{\star \star} P<0.01,{ }^{\star \star \star} P<0.001$; bars represent mean $\pm S E M ; n=6$ for each group; Six sections per animal were selected for counting.

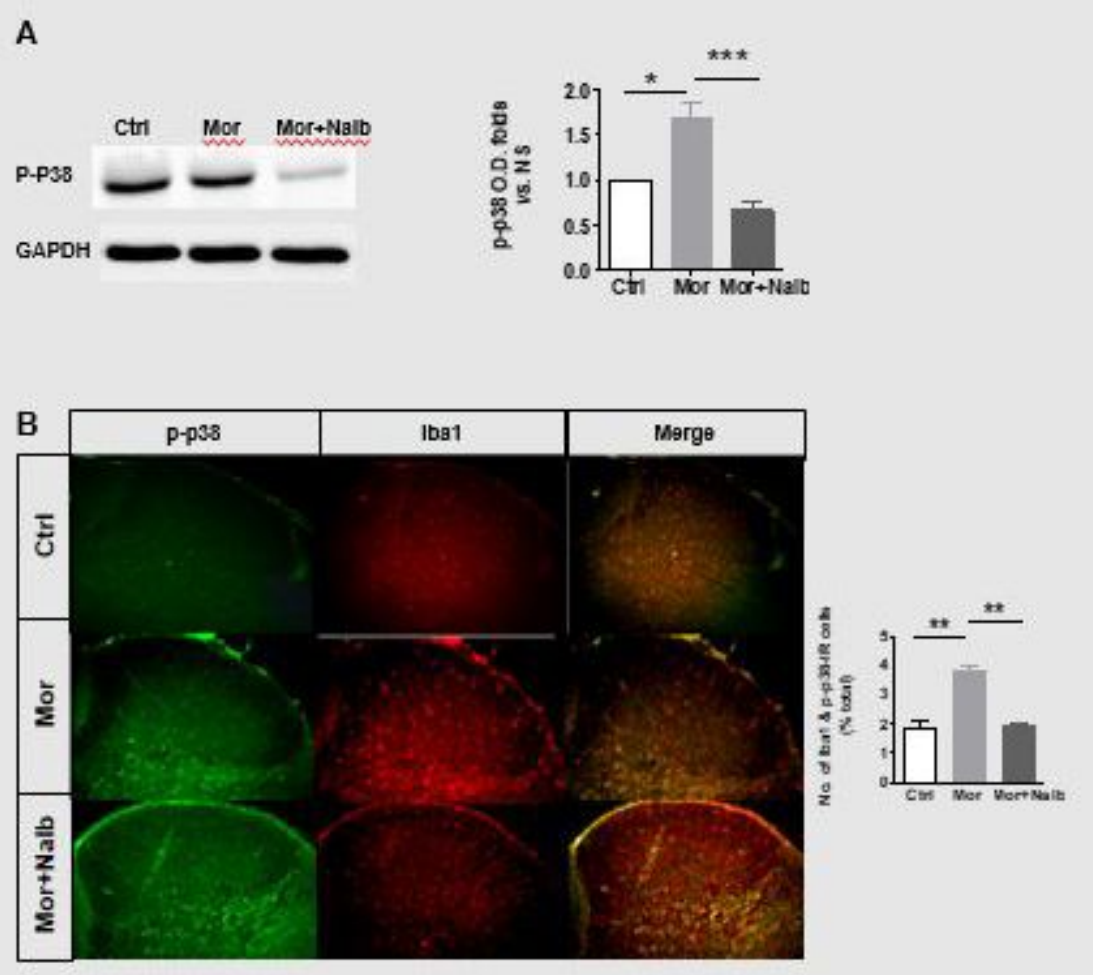

Figure 5

-P38 and CD11b are co-expression in the spinal cord after treatment with nalbuphine and morphine. (A) Western blot and quantified data of p-p38 expression in spinal cord extracts from control and morphine- 
treated animals; additional animals received intrathecal nalbuphine. After nalbuphine treatment, the pp38 expression was significantly decreased compared to morphine treatment. ${ }^{*} P<0.05,{ }^{\star} * \star P<0.001$; bars represent mean $\pm S E M ; n=6$ for each group. (B) Representative images of spinal cord sections immunostained with anti-lba1 and p-p38 after morphine treatment; additional animals received concomitant treatment with nalbuphine following morphine treatment. Bar graph showing the number of p-p38 and Iba1 immunoreactive (IR) cells per spinal cord section from animals with treatments indicated before. The Iba $1+$ and p-p38+ cells were significantly increased in morphine i.t. injection treatment, while decreased after nalbuphine administration. ${ }^{*} \mathrm{P}<0.01$; bars represent mean $\pm S E M ; n=6$ for each group; Six sections per animal were selected for counting. 